\title{
Diagnostic utility of haematological parameters and C-reactive protein in early detection of neonatal septicaemia in developing countries
}

\author{
Rakshatha Nayak ${ }^{1}$, Subhan Ali R ${ }^{2, *}$, Nisha T.G ${ }^{3}$ \\ ${ }^{1}$ PG Student, ${ }^{2,3}$ Assistant Professor, Dept. of Pathology, Yenepoya Medical College, Mangalore, Karnataka, India \\ *Corresponding Author: \\ Email: subhanalir@gmail.com
}

\begin{abstract}
Introduction: Septicaemia is one of the major causes of mortality and morbidity during the neonatal period especially in developing countries. Early diagnosis of neonatal sepsis is a diagnostic problem due to non-specific clinical features. Blood culture is the gold standard, but it takes several days and is expensive. Hence there is a critical need for laboratory tests that aid in the rapid diagnosis of neonatal sepsis.

Aims and Objectives: To measure the role of Haematological Scoring System and C-reactive protein as an early diagnostic tool for neonatal sepsis.

Materials and Methods: This was a prospective study performed on 116 neonates, who were clinically suspected cases of septicaemia, admitted at Neonatal Intensive Care Unit, Yenepoya Hospital, Mangalore. Each hematologic parameter like total leukocyte count, polymorphonuclear neutrophil (PMN) count, immature PMN count, platelet count etc. were analysed in all cases using the Haematological Scoring System (HSS) formulated by Rodwell et al. Blood cultures were performed as a gold standard. C-reactive protein (CRP) was also estimated.

Results: Out of 116 neonates, 61(52\%) were male neonates and 55(48\%) were female neonates. $18(15 \%)$ cases were with proven sepsis, 58(50\%) cases with probable sepsis and 40(35\%) cases were on the safer side. The HSS was found to have a sensitivity of $94.4 \%$, specificity of $75.5 \%$, positive predictive value was $41.4 \%$ and negative predictive value was $98.6 \%$.

Conclusions: HSS is a rapid, simple and easily affordable screening tool for early detection of neonatal septicaemia. It also provides an effective guideline to make decisions regarding judicious use of antibiotics.
\end{abstract}

Keywords: Blood culture, Haematological Scoring System, Neonates, Septicaemia, Rapid diagnosis.

Received: $16^{\text {th }}$ April, 2017

Accepted: $27^{\text {th }}$ September, 2017

\section{Introduction}

Neonatal septicaemia is a systemic infection seen in the infants before 28 days of life. ${ }^{1}$ Nearly one million deaths per year are caused by neonatal sepsis, accounting for over $25 \%$ of global neonatal deaths. Majority of these deaths occur in developing countries. ${ }^{2}$ As per National Neonatal Perinatal Database (NNPD) 2002-2003, the incidence of neonatal sepsis in India was 30 per 1000 live birth. ${ }^{3}$

The protection provided by effective barriers like placenta and amniotic sac gets exposed to the microbial world during birth. ${ }^{4}$ The most common organism is Klebsiella pneumonia followed by Staphylococcus aureus and Pseudomonas in India. ${ }^{5}$ Isolation of the causative microorganisms by using blood culture is the gold standard method for diagnosis, but it takes 24-72 hrs for obtaining the reports and in some cases possibility of false negative result may be present. ${ }^{6,7}$ Recently various markers which are both sensitive and specific have been evaluated but they are expensive and sophisticated. ${ }^{8}$ Hence rapid and cost effective methods like Haematological Scoring System (HSS) that includes white blood cell count, absolute neutrophil count, platelet count and C-reactive protein(CRP) estimation have been evaluated in this study to know its diagnostic role in developing countries. ${ }^{9}$

\section{Materials and Methods}

This was a prospective study performed on 116 neonates, who were clinically suspected cases of septicaemia or with predisposing perinatal factors, admitted at Neonatal Intensive Care Unit of our institution. Detailed history was taken and the information was recorded in a specifically designed profoma. The neonates were divided into 3 groups. (Table 1)

The collection of blood samples were done by peripheral venepuncture in an ethylene diamine tetra acetic acid (EDTA) vial. The total WBC counts were counted on a Sysmex counter (Sysmex XS 1000i) and corrected for nucleated red blood cells. Differential counts were performed on blood smears stained by Leishman stain, counting 100 cells. All smears were reviewed by a consultant pathologist blinded to the infection status of the infants. The 
haematological findings were analyzed according to the Haematological Scoring System (HSS) of Rodwell et al. ${ }^{10}$ The HSS assigns a score of one for each of the seven criteria found to be significantly associated with sepsis like total WBC count, total polymorphonuclear (PMN) leucocyte count, Immature:total (I:T) PMN count etc (Table 2) with one exception. An abnormal total PMN count is assigned a score of 2 instead of 1 , if no mature are seen on the peripheral smear to compensate for the low immature:mature (I:M) PMN ratio. Immature neutrophils include promyelocyte, myelocyte, metamyelocytes, and band form. (Fig.1a, b) Degenerative changes in neutrophils include vacuolization (Fig. 1c), toxic granulations, and Dohle bodies. Interpretation is shown in Table 3. Minimum score is 0 and maximum score is 8 . Blood cultures were performed as a gold standard. CRP was also estimated. Nucleated red blood cells (NRBC) were counted in each case. Sensitivity, specificity, and positive and negative predictive values (PPVs and NPVs) were evaluated for each of the individual criteria and for the overall score of HSS and for CRP using standard statistical methods. $\mathrm{P}$ value $<0.05$ was considered as statistically significant. ${ }^{14}$

Inclusion Criteria: All clinically suspected cases of neonatal sepsis were included in the study.

Exclusion Criteria: Neonates of mothers with diabetes, pregnancy induced hypertension and neonates with history of asphyxia and congenital anomalies, prematurity (<30 weeks), blood group incompatibility, any intake of previous antibiotic were excluded by a detailed perinatal history and clinical examination.

Table 1: Categorization of study population.

\begin{tabular}{|c|l|}
\hline Groups & \multicolumn{1}{c|}{ Presentation } \\
\hline 1 & Sepsis with positive blood culture \\
\hline 2 & $\begin{array}{l}\text { Probable sepsis with strong clinical history } \\
\text { but negative blood culture }\end{array}$ \\
\hline 3 & $\begin{array}{l}\text { Normal infants with minimal evidence of } \\
\text { sepsis }\end{array}$ \\
\hline
\end{tabular}

Table 2: Haematological scoring system

\begin{tabular}{|l|l|c|}
\hline \multicolumn{1}{|c|}{ Criteria } & \multicolumn{1}{c|}{ Abnormality } & Score \\
\hline \multirow{3}{*}{$\begin{array}{l}\text { Total WBC } \\
\text { Count }\end{array}$} & $\leq 5000 /$ cumm & \multirow{2}{*}{1} \\
\cline { 2 - 2 } & $\geq 25,000$ at birth & \multirow{2}{*}{} \\
\cline { 2 - 2 } & $\geq 30,000$ at $12-24$ hours \\
\cline { 2 - 2 } & $>21,000$-Day 2 onwards & \\
\hline Total PMN Count & No mature PMN & 1 \\
\cline { 2 - 2 } & Increased (>5,400/cumm) & 1 \\
\hline $\begin{array}{l}\text { Immature PMN } \\
\text { Count }\end{array}$ & Increased $(\geq 600 /$ cumm) & 1 \\
\hline I:T PMN Ratio & Increased $(\geq 0.12)$ & 1 \\
\hline I:M PMN Ratio & Increased( $\geq 0.3)$ & 1 \\
\hline $\begin{array}{l}\text { Degenerative } \\
\text { Changes In PMN }\end{array}$ & $\begin{array}{l}\text { Toxic granules/ cytoplasmic } \\
\text { vacuoles }\end{array}$ & 1 \\
\hline Platelet Count & $\leq 1,50,000 /$ cumm & 1 \\
\hline
\end{tabular}

Table 3: Interpretation of Haematological Scoring System

\begin{tabular}{|l|l|}
\hline \multicolumn{1}{|c|}{ Score } & \multicolumn{1}{c|}{ Interpretation } \\
\hline$\leq 2$ & Sepsis is very unlikely \\
\hline 3 or 4 & Probable sepsis \\
\hline$>5$ & Sepsis or infection is very likely \\
\hline
\end{tabular}



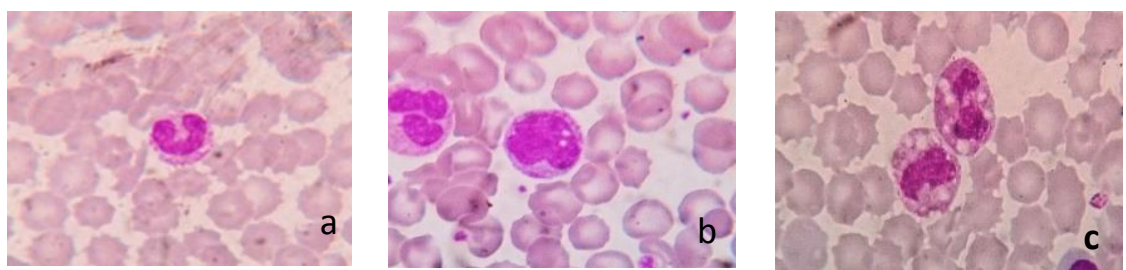

Fig.1: Photomicrograph of peripheral smear showing: a) and b): Immature neutrophils; c) Neutrophils with cytoplasmic vacuoles

\section{Result}

Out of 116 neonates who were clinically suspected of sepsis, 61(52\%) were male and $55(48 \%)$ were female neonates, with Male: Female ratio of 1.1:1. Maximum number (68; $58.6 \%)$ of patients belonged to the age group 113 days. Maximum cases $(42 \%)$ belonged to low birth weight group of 1 to $<2.5 \mathrm{~kg}$. $18(15 \%)$ cases were with proven sepsis, 58(50\%) cases with probable sepsis and 40(35\%) cases were on the safer side. Escherischia coli was the most common organism isolated by blood culture followed by Staphylococcus aureus and Enterobacter spp.

Table 4 shows scores of each group. 17 (94.4\%) neonates with proven sepsis had scores $>=5.1(5.6 \%)$ neonates with proven sepsis had scores 3-4. 21 (36.2\%) neonates with history of probable infection had scores $>=5$ and $29(50 \%)$ had scores 3-4 suggesting the possibility of sepsis. $3(7.5 \%)$ of normal neonates had score $>=5$ and $7(17.5 \%)$ had scores $3-4$ suggesting the possibility of sepsis in these cases. $20(50 \%)$ of normal neonates and $8(13.7 \%)$ neonates with probable infection had score $<=2$, which implies sepsis was unlikely in these cases.

In this study, (Table 5) individual parameters like I:T PMN ratio and Immature PMN counts showed high sensitivity (100\%). Total WBC count $(83.6 \%)$ was highly specific followed by platelet count (78.5\%) in identifying neonates with sepsis. Negative predictive value was high in I:T PMN ratio and Immature PMN count (100\%) followed by HSS(98.6\%) which was useful in identifying neonates that did not have any evidence of sepsis. The HSS was found to have a sensitivity of $(94.4 \%)$, specificity of (75.5\%), positive predictive value was $(41.4 \%)$ and negative predictive value was (98.6\%). CRP provides limited sensitivity (44.4\%) but provides very high negative predictive values (87.9\%) and is thus useful for identifying infants unlikely to be infected. Nucleated Red blood cells values were higher in sepsis and significantly seen in $70 \%$ of cases in proven sepsis.

Table 4: Distribution of cases according to sepsis score

\begin{tabular}{|l|c|c|c|}
\hline \multicolumn{1}{|c|}{ Category } & Scores 0-2 & Scores 3-4 & Scores $>=5$ \\
\hline Proven sepsis (18) & 0 & 1 & 17 \\
\hline Probable sepsis (58) & 8 & 29 & 21 \\
\hline No sepsis (40) & 20 & 17 & 3 \\
\hline Total (116) & 28 & 47 & 41 \\
\hline
\end{tabular}

Table 5: Performance of individual haematological parameter \& CRP

\begin{tabular}{|l|c|c|c|c|}
\hline \multicolumn{1}{|c|}{ Criteria } & $\begin{array}{c}\text { Sensitivity } \\
(\boldsymbol{\%})\end{array}$ & $\begin{array}{c}\text { Specificity } \\
(\mathbf{\%})\end{array}$ & $\begin{array}{c}\text { Positive } \\
\text { Predictive } \\
\text { Value (\%) }\end{array}$ & $\begin{array}{c}\text { Negative } \\
\text { Predictive } \\
\text { Value (\%) }\end{array}$ \\
\hline HSS & 94.4 & 75.5 & 41.4 & 98.6 \\
\hline $\begin{array}{l}\text { Total WBC } \\
\text { Count }\end{array}$ & 38.8 & 83.6 & 30.4 & 88.1 \\
\hline $\begin{array}{l}\text { Total PMN } \\
\text { Count }\end{array}$ & 83.3 & 33.6 & 18.7 & 91.6 \\
\hline $\begin{array}{l}\text { Immature } \\
\text { PMN Count }\end{array}$ & 100 & 24.4 & 19.5 & 100 \\
\hline I:T PMN Ratio & 100 & 27.5 & 20.2 & 100 \\
\hline $\begin{array}{l}\text { I:M PMN } \\
\text { Ratio }\end{array}$ & 72.2 & 69.3 & 30.2 & 93.1 \\
\hline Platelet Count & 66.6 & 78.5 & 36.3 & 92.7 \\
\hline CRP & 44.4 & 74.4 & 24.2 & 87.9 \\
\hline
\end{tabular}




\section{Discussion}

In developing countries like India, neonatal sepsis remains a problematic disease and is potentially life-threatening. Some of the risk factors for sepsis include maternal factors like premature rupture of membranes, maternal infections, procedures etc. and infant factors like low birth weight, poor cord care, congenital anomalies, low APGAR score etc. ${ }^{1,2,4}$ Clinically the patients may present with respiratory distress, irritability, hypothermia, hypo or hyperglycaemia, vomiting, poor feeding, cyanotic spells, seizures and shock. ${ }^{6}$ It is a great challenge to diagnose neonatal septicaemia as the early signs of sepsis may be subtle and different at different gestational ages. ${ }^{11}$ The evaluation of screening tests for neonatal sepsis is an absolute necessity as the infection may present a serious threat to the baby. Non infected neonates should also be identified to avoid unnecessary antibiotic administration and emergence of resistant microorganisms. ${ }^{12,14-16}$

An ideal screening test should have high sensitivity and high negative predictive value that would detect all the patients with the disease and exclude all the cases, which do not have that specific disease. The risk of missing a patient with a certain infection is greater than the risk of over treatment with antibiotics hence a low specificity and positive predictive value are acceptable. ${ }^{6}$ Even though blood culture is gold standard test for diagnosing sepsis, the procedure is expensive, time consuming as it takes $48-72$ hours, has low yield due to insufficient sample volumes, intermittent or low-density bacteraemia, or suppression of bacterial growth by earlier (i.e., intrapartum) antibiotic administration. ${ }^{17-19}$ Moreover the technique demands a well-equipped laboratory which is not available in most of the community hospitals. ${ }^{20-22}$

In the recent past, various markers which are both sensitive and specific like ELISA methods, prolactins, haptoglobins, interleukins, molecular analysis by polymerase chain reaction (PCR) for bacterial DNA etc. to diagnose neonatal sepsis have been evaluated but they are expensive, sophisticated and unavailable in rural setups. ${ }^{1,8,13}$

A Hematologic Scoring System (HSS) of Rodwell was introduced for early diagnosis of neonatal septicaemia which is easy to perform and applicable to all infants, including those who have received treatment. ${ }^{9,10}$ It included haematological parameters and showed that such score could accurately predict the presence or absence of infection and was reliable.

In our study, among the infected newborns, the predominance of male was seen. The factors that regulate the synthesis of alpha globulin are situated on the $\mathrm{X}$ chromosome. Males have only one $\mathrm{X}$ chromosome; hence are less immunologically protected than the females. ${ }^{9}$ This is consistent with other studies. . $^{3-9,14-16}$

In this study, individual parameters like I:T PMN ratio and Immature PMN counts showed high sensitivity and negative predictive value which was consistent with many other studies.(Table 6)

Table 6: Comparison of sensitivity and NPV of individual parameters with other studies

\begin{tabular}{|l|l|}
\hline \multicolumn{1}{|c|}{ Series } & \multicolumn{1}{c|}{ Highest sensitivity and NPV } \\
\hline Nayana NS et al & I:T ratio \\
\hline Supreetha MS et al & I:M ratio \\
\hline Narasimha A et al & Total PMN count \\
\hline Majumdar A et al & I:T ratio, I:M ratio \\
\hline Meirina F et al & I:T ratio, total PMN count \\
\hline Khair KB et al & I:T ratio, I:M ratio \\
\hline Present study & I:T ratio and Immature PMN counts \\
\hline
\end{tabular}

The certainty of sepsis increases as the score increases. HSS score of more $>3$ was considered significant by various studies. ${ }^{3-9}$ A score $\leq 2$ suggested that sepsis was unlikely. In the present study, HSS was significantly $\quad(\mathrm{P}<0.005)$ associated with sepsis. A score of $>=5$ was found to have a sensitivity of $94.4 \%$, specificity of $75.5 \%$, positive predictive value was $41.4 \%$ and negative predictive value was $98.6 \%$.

HSS can be considered a superior and more reliable screening tool for sepsis than any of the individual haematological parameter due to higher specificity and positive predictive value. It simplifies the interpretation of haematological profiles.

Nucleated Red blood cells values were higher in sepsis and significantly seen in $70 \%$ of cases in proven sepsis. This was consistent with other studies. ${ }^{5}$ Studies showed that mean NRBC value of 10 is seen in term and healthy neonates. ${ }^{23}$ NRBC count greater than the indicated reference values may be seen in postnatal hypoxia, anaemia, maternal diabetes or acute stress and sometimes in neonatal 
septicaemia. In sepsis, cytokines are released which play an important role in stimulating nucleated RBC production independent of hypoxia. $^{5}$

C-reactive protein which is released in sepsis is a non-specific acute phase protein. Elevated CRP levels are highly suggestive of Neonatal sepsis. ${ }^{3,6-9}$ CRP measurement can be done in conjunction with other diagnostic tests such as HSS and blood culture to establish or exclude the diagnosis of sepsis in neonates. ${ }^{13,15}$ In the present study CRP $>10 \mathrm{mg} / \mathrm{L}$ was the cut of value to suspect neonatal sepsis. ${ }^{8}$ Values of sensitivity \& specificity of CRP were $44.4 \%$ and $74.4 \%$ respectively. PPV of CRP was $24.2 \%$ and NPV of CRP was $87.9 \%$.CRP provides high negative predictive values and hence plays an important role in identifying infants unlikely to be infected or for monitoring the response to treatment.

\section{Conclusion}

HSS is a rapid, easy to perform, cost effective and easily available screening system for early detection of neonatal septicaemia. It also provides an effective guideline to make decisions regarding judicious use of antibiotics. Combining it with CRP levels gives a good analysis of sepsis. It has a good sensitivity, rather than high specificity and a good negative predictive value and can therefore help in safely withholding antibiotic administration in noninfected infants. Peripheral blood showing elevated nucleated RBCs values are significantly associated with newborn sepsis and could be an interesting marker of neonatal sepsis in absence of hypoxia.

\section{References}

1. Simonsen KA, Anderson-Berry AL, Delair SL, Davies HD. Early-Onset Neonatal Sepsis. Clinical microbiological reviews 2014 Jan;27:2147.

2. Jajoo M, Kapoor K, Garg LK, Manchanda V, Mittal SK. To study the incidence and risk factors of early onset neonatal sepsis in an out born neonatal intensive care unit of India. J Clin Neonatol 2015;4:91-5.

3. Nayana NS, Sreenivas N. Neonatal Septicemia- A Smooth Technique of Diagnosis in Developing Countries. Int J Res Med Sci 2016;4:2305-8.

4. Woldu MA, Guta MB, Lenjisa JL, Tegegne GT, Tesafye G. Assessment of the Incidence of Neonatal Sepsis, its Risk Factors, Antimicrobials Use and Clinical Outcomes in Bishoftu General Hospital, Neonatal Intensive Care Unit, Debrezeit-Ethiopia. Pediat Therapeut 2014;4:214.

5. Supreetha MS, Alva SR, Shivendra VS, Kariappa TM. Evaluation of Neonatal Septicaemia Using
Hematological Parameters. International Journal of Recent Scientific Research 2015;6(2):2775-8.

6. Arif S, Ehsan A, Arif M, Hussain J, Bano R. Early diagnosis of neonatal sepsis through hametological and biochemical markers. Gomal J Med Sci 2012;11:178-82.

7. Sucilathangam G, Amuthavalli K, Velvizhi G, Ashihabegum MA, Jeyamurugan T, Palaniappan N. Early Diagnostic Markers for Neonatal Sepsis: Comparing Procalcitonin (PCT) and $\mathrm{C}$ - reactive protein (CRP). Journal of Clinical and Diagnostic Research. 2012 May;6(4):627-31.

8. Usha P, Sekhar Reddy VVACH, Uma P, Bhagya Lakshmi A. Clinical correlation of neonatal and maternal hematological parameters as predictors of neonatal sepsis. IJRRMS 2015;5(1):1-7.

9. Saleem M, Shah KI, Cheema SM, Azam M. Hematological Scoring System for Early Diagnosis of Neonatal Sepsis. JRMC 2014; 18(1):68-72.

10. Rodwell RL, Leslie AL, Tudehope DI. Early diagnosis of neonatal sepsis using a hematologic scoring system. J Pediatr 1988;112:761-7.

11. Narasimha A, Kumar MLH. Significance of Hematological Scoring System (HSS) in Early Diagnosis of Neonatal Sepsis. Indian J Hematol Blood Transfus 2011;27(1):14-7.

12. Das B, Das A, Saikia M. A study of haematological parameters in neonatal septicaemia. Indian Journal of Basic and Applied Medical Research; 2016;5(2):491-500.

13. Mondal SK, Nag DR, Banyopadhyay R, Chakraborty D, Sinha SK. Neonatal sepsis - Role of certain immunohematologic tests in rapid diagnosis. J Acad Med Sci 2012;2:97-100.

14. Majumdar A, Jana A, Jana A, Biswas S, Bhattacharyya S. Hematologic scoring system (HSS): A guide to decide judicious use of antibiotics in neonatal septicemia in developing countries. J Appl Hematol 2013;4:110-3.

15. Duhan A, Berwal A, Raikwar P, Punia A, Beniwal K, Kamra HT. Utility of Hematological Parameters in Detection of Neonatal Sepsis. JKIMSU 2016 July-September;5:100-6.

16. Shirazi H, Riaz S, Tahir R. Role of the Hematological Profile in Early Diagnosis of Neonatal Sepsis. Ann. Pak. Inst. Med. Sci. 2010;6(3):152-6.

17. Meirina F, Lubis B, Sembiring T, Rosdiana N, Siregar O.R. Hematological scoring system as an early diagnostic tool for neonatal sepsis. Paediatr Indones 2015 November;55(6):315-21.

18. El-Sonbaty MM, AlSharany W, Youness ER, Mohamed NA, Abdel-Hamid TA, Abdel-Razek ARA. Diagnostic utility of biomarkers in diagnosis of early stages of neonatal sepsis in neonatal intensive care unit in Egypt. Egyptian Pediatric Association Gazette 2016;64:91-6.

19. Pal K,Samanta AK. Evaluation of Hematological Parameters In Early Onset Neonatal Sepsis. NJIRM 2013;4(6):29-34.

20. Khair KB, Rahman MA, Sultana T, Roy CK, Rahman MQ, Shahidullah M, Ahmed ANN. Role of Hematologic Scoring System in Early Diagnosis of Neonatal Septicemia. BSMMU J 2010;3(2):62-7.

21. Bhat AA, Shamim A, Gul S, Akther R, Bhat I. Neonatal sepsis - Early detection comparing 
Procalcitonin and CRP as markers and newer tools. Int J Med and Dent Sci 2016;5(1):1048-55.

22. Hisamuddin E, Hisam A, Wahid S, Raza G.

Validity of C-reactive protein (CRP) for diagnosis of neonatal sepsis. Pak J Med Sci 2015;31(3):527-31.

23. Dhanamjayaraoteeda, Jalagam RP. A Study of Peripheral Smears in Neonates. IOSR-JDMS 2015 Feb;14(2):55-64. 